\title{
Hypertrophic osteoarthropathy (HOA) in a child with congenital cyanotic heart disease - a case report
}

\author{
Michelo S Choopa ${ }^{1 *}$, Belinda Mitchel1², Ignatius T Menyatsoe ${ }^{3}$ and Gertruida van Biljon ${ }^{4}$ \\ ${ }^{1}$ FCPaed (SA), Fellow in-training in Paediatric Nephrology, Paediatric Nephrology Unit, Department of Paediatrics and Child Health, University of Pretoria, Steve \\ Biko Academic Hospital, Pretoria, Gauteng, South Africa \\ ${ }^{2}$ FCPaed (SA), Cert Paed Cardiology (SA), Consultant Paediatric Cardiologist, Paediatric Cardiology Unit, Department of Paediatrics and Child Health, University \\ of Pretoria, Steve Biko Academic Hospital, Pretoria, Gauteng, South Africa \\ ${ }^{3}$ Radiology Registrar, Department of Radiology, University of Pretoria, Steve Biko Academic Hospital, Pretoria, Gauteng, South Africa \\ ${ }^{4}$ FCPaed (SA), Cert Paed Nephrology (SA), Professor and Consultant Paediatric Nephrologist, Paediatric Nephrology Unit, Department of Paediatrics and Child \\ Health, University of Pretoria, Steve Biko Academic Hospital, Pretoria, Gauteng, South Africa
}

\begin{abstract}
This is a case of a 12 year old girl who was diagnosed at birth with a double outlet right ventricle, pulmonary atresia, and interrupted inferior vena cava (IVC), with suspected Heterotaxy syndrome. A modified Blalock-Taussig shunt was done. She remained cyanotic due to a hypoplastic pulmonary artery bed and was considered unsuitable for further surgical intervention and was followed up at the Paediatric Cardiology clinic. At follow up at the age of 5 years old, cardiac catheterization to assess pulmonary arteries for possible surgery was conducted and she was incidentally found to have a dilated non-obstructed left renal pelvis. She was also found to be hypertensive and was treated with an angiotensin converting-enzyme inhibitor and a calcium channel blocker. Other treatment given for her cardiac disease included furosemide, digoxin, ferrous gluconate, and potassium supplementation.

She developed progressive swelling and pain of her large joints from the age of 11 years old, and immune-mediated and destructive arthropathies were excluded. She was treated with a non-steroidal anti-inflammatory drug (NSAID). The swelling and pain of her large joints did not improve and radiologic investigations were found to be in keeping with secondary hypertrophic osteoarthropathy.

In our patient, surgery was not possible for her heart condition and only conservative symptomatic treatment was offered. Her treatment remained a therapeutic challenge in view of the poly-pharmacy required to treat her multi-systemic condition. The side effects of these drugs are particularly undesirable in our patient, but had to be weighed against the poor quality of life she would have had if her treatment was focussed on her heart condition only.
\end{abstract}

\section{Introduction}

A 12 year old female patient, known to the Paediatric Cardiology unit of an academic hospital, presented with oedema, rigours abdominal pain and arthralgia of her large joints. There was no fever, haematuria or dysuria.

She was diagnosed at birth with double outlet right ventricle, pulmonary atresia, and interrupted inferior vena cava (IVC), with suspected Heterotaxy syndrome. A modified Blalock-Taussig shunt was done. She remained cyanotic due to a hypoplastic pulmonary artery bed and was considered unsuitable for further surgical intervention. She was managed conservatively with furosemide, digoxin, ferrous gluconate, and potassium supplementation.

Cardiac catheterization to assess pulmonary artery growth at the age of four years incidentally revealed a dilated left renal pelvis. Dynamic imaging revealed a dilated, non-obstructed left renal pelvis that required no surgical intervention.

She remained relatively asymptomatic despite severe central cyanosis and intermittent episodes of oedema. At the age of 5 years she developed systemic hypertension and an angiotensin- converting enzyme inhibitor and calcium channel blocker were added to her treatment.
On examination she was a well-grown girl with normal anthropometry. She was afebrile but had intermittent rigors. She had central cyanosis, digital clubbing and tender swollen knees, elbows and ankles, with decreased range of movement. Her blood pressure was normal. Her pulse rate was 120/minute. Auscultation of the heart revealed a loud second heart sound and a third heart sound. There were no signs of cardiac failure. She had hepatomegaly, but no other organomegaly or ascites. Examination of the other systems, including the neurological system was normal.

Investigations revealed a haemoglobin of $19.9 \mathrm{~g} / \mathrm{dL}(10.3-15.5 \mathrm{~g} /$ $\mathrm{dL})$, haematocrit of $63 \cdot 1 \%$ (31-44\%), total white cell count of $6 \cdot 32 \times 10^{9}$ (3.9-10.2 $\left.\times 10^{9}\right)$, serum creatinine of $45 \mathrm{micromol} / \mathrm{L}(37-63 \mu \mathrm{mol} / \mathrm{L})$, C-reactive protein of $155 \mathrm{mg} / \mathrm{L}(0-8 \mathrm{mg} / \mathrm{L})$ and procalcitonin of $<$

Correspondence to: Michelo S Choopa, FCPaed (SA), Fellow in-training in Paediatric Nephrology, Paediatric Nephrology Unit, Department of Paediatrics and Child Health, University of Pretoria, Steve Biko Academic Hospital, Pretoria, Gauteng, South Africa, Tel: +27123545276; Fax: +27123545275; E-mail: mchoopa@yahoo.co.uk

Key words: hypertrophic osteoarthropathy, congenital cyanotic heart disease

Received: June 28, 2016; Accepted: July 27, 2016; Published: July 30, 2016 
$0 \cdot 1 \mu \mathrm{g} / \mathrm{L}(2-4 \mu \mathrm{g} / \mathrm{L})$. Urine dipstick test and culture were negative. Radiological and serological investigations for rheumatoid arthritis were negative. Renal sonar showed bilateral poor cortico-medullary differentiation, severe hydronephrosis of the left kidney, mild hydronephrosis of the right kidney and a large stone with scattered gas echoes but no abscesses in the left renal pelvis. An uncontrasted CTscan of the abdomen showed polysplenia and a staghorn stone in the left renal pelvis.

\section{Figure 1}

Tc-99m MAG3 renogram revealed split renal function of $29 \cdot 82 \%$ and $70 \cdot 18 \%$ in the left and right kidneys respectively. Cystoscopy showed a left pelvi-ureteric junction obstruction and a JJ-stent was inserted. Extracorporeal lithotripsy was considered, but was not undertaken as she would not have tolerated anaesthesia and the procedure from a cardiovascular system point of view. She received intravenous antibiotics for the suspected pyelonephritis, oral paracetamol and ibuprofen for the painful swollen joints, and the anti-hypertensive treatment was discontinued. She was discharged from hospital after three weeks, and chronic medication was continued.

She was re-admitted six weeks later with worsening arthralgia, swollen large joints and a first-onset generalised tonic-clonic seizure. Her blood pressure was normal. Contrasted computed tomographic scan of the brain revealed an old right cerebellar infarct. On the electroencephalogram, there was diffuse slowing over the right hemisphere with focal epileptiform activity over the right mid-temporal region. Carbamazepine was added to her treatment. X-rays of long bones showed periosteal reaction. A diagnosis of secondary hypertrophic osteoarthropathy (HOA) was made. This girl developed cerebral infarcts, epilepsy and secondary hypertrophic osteoarthropathy which are all known complications of longstanding inoperable cyanotic congenital heart disease.

\section{Figure 2}

\section{Discussion}

HOA is characterized by a triad of arthritis, digital clubbing and new bone formation with periosteal inflammation [1]. Factors included in the pathogenesis are circulating vasodilators, tissue hypoxia, neural mechanisms and genetic factors [2].

HOA develops in children with cyanotic congenital heart disease (CHD) who have decreased pulmonary blood flow causing persisting severe central cyanosis $[3,4]$. Cyanosis and digital clubbing usually resolve after corrective cardiac surgery [5]. In our patient, surgery

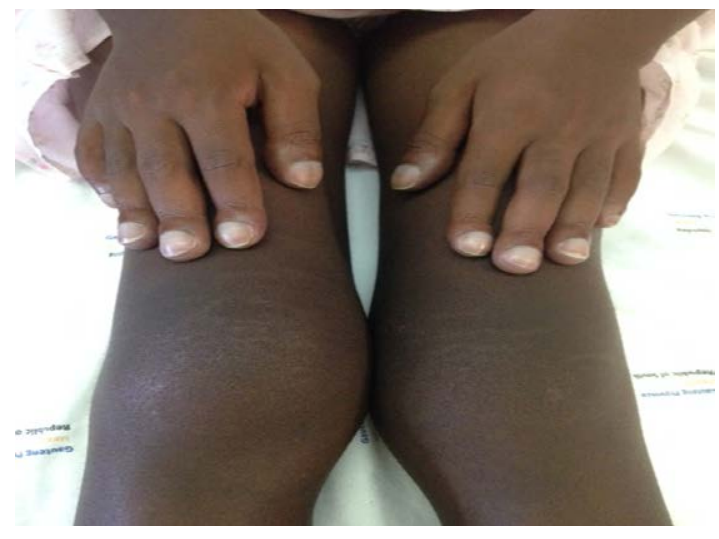

Figure 1. Digital clubbing and swelling of knees.

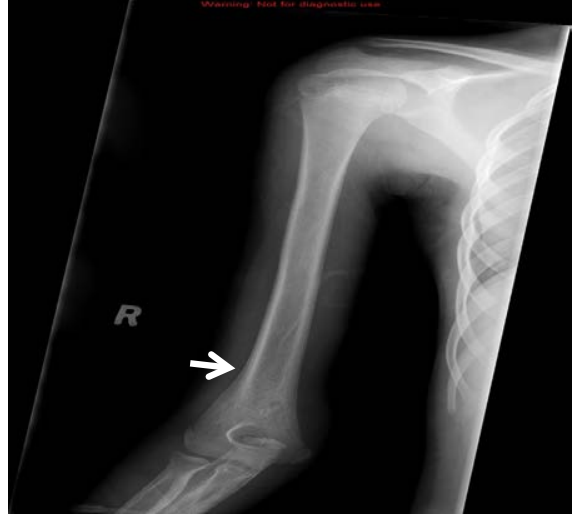

Figure 2. Lamellation of distal right humerus (white arrow).

was not possible and only conservative symptomatic treatment was offered. Her pain has been controlled with a non-steroidal antiinflammatory drug (NSAID). The side effects of NSAIDs include fluid retention, worsening of oedema, hypertension, heart failure and fatal thromboembolism. All these side effects are particularly undesirable in our patient, but have to be weighed against the poor quality of life she will have if her treatment is focussed on her heart condition only.

\section{Authorship and contributorship}

MS Choopa conducted the literature search and wrote up the manuscript; B Mitchell and $G$ van Biljon assisted in editing and writing of the manuscript; and IT Menyatsoe assisted in the interpretation of the radiological images.

\section{Financial assistance}

None

\section{Declaration of interest}

The authors have nothing to disclose.

\section{Consent}

This was obtained from the patient and parent for photographs and publication.

\section{References}

1. Ray D, Menon RK, Menon PSN, Gupta G, Shrivastava S, et al. (1988) Hypertrophic osteoarthropathy, tuberculoma of brain and cyanotic heart disease. Indian J Pediatr 55: 304-307. [Crossref]

2. Shneerson JM (1981) Digital clubbing and hypertrophic osteoarthropathy: The underlying mechanism. Br J Dis Chest 75: 113-131. [Crossref]

3. Ansell BM (1991) Hypertrophic osteoarthropathy in the paediatric age. Clinical and Experimental Rheumatology [e-journal] 10:15-18.

4. Martinez-Lavin M, Bobadilla M, Casanova J, Attié F, Martinez M (1982) Hypertrophic osteoarthropathy in cyanotic congenital heart disease: its prevalence and relationship to bypass of the lung. Arthritis Rheum 25: 1186-93. [Crossref]

5. Frand M, Koren G, Rubinstein Z (198) Reversible hypertrophic osteoarthropathy associated with cyanotic congenital heart disease. Am J Dis Child 136: 687-689. [Crossref]

Copyright: (C2016 Choopa MS. This is an open-access article distributed under the terms of the Creative Commons Attribution License, which permits unrestricted use, distribution, and reproduction in any medium, provided the original author and source are credited. 